\title{
Towards a Dynamic Theory of Belief-Sharing in Cooperative Dialogues
}

\author{
Hisashi Komatsu \\ Norihiro Ogata \\ Akira Ishikawa \\ The Toin Corporation University of Tsukuba Sophia University
}

\section{Introduction}

In this paper, we propose a dynamic theory of beliefsharing which deals with certain processes of forming and revising shared beliefs during cooperative dialogues.

Since Clark \& Marshall(1981) the problem of determination of the referents of referring expressions has been discussed in relation to mutual knowledge. In natural language processing, there have also been several studies treating this problem of referent-determination in terms of mutual knowledge (Perrault \& Cohen, 1981; Joshi, 1982; Nadathur \& Joshi, 1983; Appelt, 1985). In this paper, we conceive referent-determination as a process of beliefsharing in dialogues, and propose a formal theory of dialogue in which referent-determination can be characterized as part of belief-sharing processes. We use Discourse Representation Theory (DRT) to model the characteristics of referents in discourse (Kamp, 1981, 1990; Asher, 1993), and propose a model of dynamic maintenance of the mutual beliefs of the participants in dialogues based on Clause Maintenance System (CMS) (Doyle, 1979; Levesque, 1989; Poole, 1988; Reggia, 1983; de Klcer, 1986; Reiter \& de Kleer, 1987). By this model, we characterize the relationships between a dialogue process and its successfulness, which is mainly illustrated by examples of referent-determination but can be applied to any type of belief-sharing.

\section{Dynamic Maintenance of Shared Beliefs}

\section{$2.1 \quad$ DRS}

However cooperative, real-world dialogues are fraught with hedges, understatements, or even white lies, which would necessitate introducing a distinction between what is literally conveyed by an utterance, and its real intent on the part of both speaker and hearer. In this study, however, we restrict ourselves to those cases without such complications, and assume that an utterance reflects the speaker's intent in a straight manner, and is taken as such by the hearer. The con- tent of an utterance is represented in the following style:

(1)

$K:$\begin{tabular}{l|}
\hline$a, b, x, y, z, \ldots$ \\
\hline $\operatorname{Bel}(a, K)$ \\
$\operatorname{Bel}(b, K)$ \\
\hline$A(x), B(y), C(z), \ldots$ \\
\end{tabular}

We call $K$ discourse representation structure (DRS), $\{a, b, x, y, z, \ldots\} K$ 's domain ( $\left.\mathbf{U}_{K}\right)$, the elements of $\mathbf{U}_{K}$ discourse referents, the boxed area below the unbroken line $K$ 's condition part $\left(\mathbf{C}_{K}\right)$, and $\mathrm{C}_{K}$ 's elements conditions. $K$ is represented as $\left\langle\mathbf{U}_{K}, \mathbf{C}_{K}\right\rangle$. The broken line divides $\mathbf{C}_{K}$ into the self-reforential part SRP (above the line), and the dialogue database $\mathbf{D B}(K)$ (below the line). A condition is the result of an $n(\geq 0)$ times application of $\operatorname{Bel}(\alpha,$.$) to a first-order formula p . \operatorname{Bel}(\alpha,$.$) is$ called a belief operator, where $\alpha$ designates the utterer. Given $\phi$ as a condition, $\operatorname{Bel}(\alpha, \phi)$ reads "the participant $\alpha$ believes $\phi . " n$ is called the rank of $\phi$ with regard to its embedding within belief operators. Conditions of rank 0 are called bare formulas, while those with a rank greater than 0 belief formulas. $K$ represents the shared beliefs formed through a dialogue between the two participants $a$ and $b$. The conditions in SRP indicate a recursive embedding of self-referential belief sturucture with regard to common knowledge, and are assumed throughout the dialogue. By contrast, $\mathrm{DB}(K)$ is empty when a dialogue starts off. Thus, at the outset of a dialogue, the DRS $K_{0}=\left\langle\{a, b\},\left\{\operatorname{Bel}\left(a, K_{0}\right), \operatorname{Bel}\left(b, K_{0}\right)\right\}\right\rangle$. As an utterance is made, new discourse entitics may be introduced, making it necessary to add new conditions to $\mathrm{DB}(K)$ and sometimes to retract or negate part of the conditions in $\mathbf{D B}(K)$. With the progress of the dialogue, the DRS changes from $K_{0} \Rightarrow K_{1} \Rightarrow \ldots \Rightarrow$ $K_{n} \Rightarrow \ldots$

Since only cooperative dialogues are considered, the goal is to arrive at a DRS in which no contradictory beliefs are held by the participants. But this goal is not always achieved. We also assume that at certain points of a dialogue, the participants can hold contradictory beliefs, and that the same pariticipant 
can hold contradictory beliefs at different points of a dialogue, whereas the same participant cannot hold contradictory beliefs at any particular point.

In what follows, we just indicate $\mathbf{D B}(K)$ unless otherwise noted.

\subsection{How shared beliefs are registered}

An utterance made by a participant in a dialogue is transformed into a condition(s) and registered in DB $(K)$, following the constraints stated below.

First, discourse referents are taken to be epistemological entities withont connterparts in surface sentences, but introduced into the DRS by the participants of a dialogue, and of which properties corresponding to surface linguistic expressions are predicated. Thus, an utterance

(2) a: Sato is a student

is not analyzed as

(3) student(Sato)

but as

(4) Sato(x), student $(x)$

with the discourse referent $x$ introduced into $\mathrm{U}_{K}$ by $a$, and the predicates corresponding to expressions in the utterance.

Second, an utterance is registered not in the form of a bare formula, but in the form of a belief formula indicating the bolief agent. (4), for example, is registered as

(5) Bel $(a$, Sato $(x)), \operatorname{Bel}(a$, student $(x))$

because at (2), b has not agreed with or opposed a's utterance. Note that $(5)$ is nevertheless a shared belief at this point. Suppose (6) is uttered following upon (2):

(6) b: Yes, he is.

This ntterance is interpreted as

(7) $\operatorname{Bel}(b, \operatorname{Sato}(x)), \operatorname{Bel}(b$, student $(x))$

and so registered in $\operatorname{DB}(K)$. At this point, both (5) and (7) are shared belicfs, which nocars (4) is a beliof shared by $a$ and $b$. This transition is fomulated as the axiom of shared belicf:

(8) The axiom of shared belief

When DD $(K)$ contains $B e l(a, p)$, and $B e l(b, p)$, $\operatorname{DB}\left(K^{\prime}\right)$ obtained from $\mathrm{DB}(K)$ by the substitution of $p$ for them is equivalent to $\mathrm{DB}(K)$.

DB $(K)$ can be derived from DB $\left(K^{\prime}\right)$ without using this axiorn, since $K$ has the self-referential part SRP. But the converse does not hold. The axiom of slared belinf allows the rank of shared beliefs to be zero, while the conditions in general are initially segistered with a rank higher than zero.
Third, there is involved a step of identification in the transition from $b$ 's utterance of (6) to the conclition (7). Just as the discourse referent $x$ was intro duced by $a$ 's utterance of (2), b introduces a distinct disconrse referent $y$, in terms of which

(9) Bel(b,Sato $(y))$, Bel $(b$, student $(y))$

is registered in $\operatorname{DB}(K)$. We assume that $a$ and $b$ agree to the identity of $x$ and $y$ at this point.

To sum up, in dialogue (2), (6), DB $(K)$ is composed of (5) alone when (2) is uttered, but is extended by the utterance of $(6)$ as follows:

(10) $\operatorname{Bel}(a, x=y), \operatorname{Bel}(b, x=y)$, Bel $(a, \operatorname{Sato}(x)), \operatorname{Bel}(a, \operatorname{Sato}(y))$, $\operatorname{Bel}(b, \operatorname{Sato}(x)), \operatorname{Bel}(b, \operatorname{Sato}(y))$, Bel $(a$, student $(x)), B \operatorname{Bel}(a$, student $(y))$, Bel(b, student $(x))$, Bel $(b$, student $(y))$.

By applying the axiom of shared belief, and $x=y$, we obtain

(11) Sato(x), student $(x)$

By contrast,

(1.2) 1.r: Sato is a stuclent.

2.b: No, he is an office derk now.

can only lave its $\mathbf{D B}(K)$ reduced to

(13) Sato(x), Bel $(a$, student $(x))$, Bel $(b$, office_clerk $(x))$.

\section{Diachronic analysis of dia- logue}

In this section, we consider the changes DRS's unlergo in the course of a clialogue. In (2), (6) in the previous section, we saw a case where a DRS with nothing but slated beliefs is successfully obtained in one inning, so to speak, withont incurring any conflict. We will look at the other three kinds of cases in which conflicts are treated in particular ways which adinit of formalization in terms of CMS.

\subsection{Direct solution of conflicts}

Consider the following dialogue.

(1.4) 1. a: Sato is a good guy.

2. b: By no means, ho is a liar.

3. at No kidding.

Just after (14.2) is attered, DB $(K)$ looks as follows:

(15) $\operatorname{Bel}(a, x=y), \operatorname{Bel}(b, x=y)$, Bel $(a, \operatorname{Sato}(x)), \operatorname{Bel}(a, \operatorname{Sato}(y))$, Bel(b, Sato(x)), Bel(b, Sato(y)),

$\operatorname{Bel}(a, \operatorname{good}(x))$,

$\operatorname{Bel}(b, \operatorname{liar}(y))$. 
The utterance of (14.3) is considered as the consequence of an inference such as this:

(16) 1. $x=y$
2. $\operatorname{Sato}(x)$
3. Bel(a,good $(x))$
4. $\operatorname{Bel}(b, \operatorname{liar}(x))$

is derived from (15). (16.3-4) do not bring about an inconsistency since they are belief formulas with different propositions inside. But obviously, $a$ has drawn an inconsistency by taking off the belief operators, and carrying out the following inference.

(17) $1 . x=y$
2. $\operatorname{liar}(y)$
3. $\operatorname{liar}(x)$
4. $\forall x(\operatorname{liar}(x) \rightarrow \neg \operatorname{good}(x))$
5. $\neg \operatorname{good}(x)$
6. $\operatorname{good}(x)$
7. $\square$

Suppose (14) is continued as follows:

(18) a: I mean the Sato in the linguistics department. $b$ : Oh, I thought you were talking about the Sato in the AI department. The one you mean is indeed a good guy.

(19) a: He does sometimes. But you can't dislike him. $b$ : I guess not.

In this case, in order to avoid the conflict, one traces its causes, and retracts the weakest one (16.1) for (18), and (17.4) for (19), or replaces it by its negation. As a result, (18), for examle, is associated with

(20) $\operatorname{Bel}(a, \neg x=y)$,

$\operatorname{Bel}(a, \operatorname{Sato}(x))$,

$\operatorname{Bel}(a, \operatorname{Sato}(y))$,

$\operatorname{Bel}(b, \neg x=y)$,

$\operatorname{Bel}(b$, Sato $(x))$,

$\operatorname{Bel}(b, \operatorname{Sato}(y))$,

$\operatorname{Bel}(a, \operatorname{LiD}(x)), \operatorname{Bel}(a, \operatorname{AiD}(y))$,

$\operatorname{Bel}(a, \forall x(\operatorname{liar}(x) \rightarrow-\operatorname{good}(x)))$,

$\operatorname{Bel}(b, \operatorname{LiD}(x)), \operatorname{Bel}(b, A i D(y))$,

$\operatorname{Bel}(b, \forall x(\operatorname{liar}(x) \rightarrow \neg \operatorname{good}(x)))$,

$\operatorname{Bel}(a, \operatorname{good}(x)), \operatorname{Bel}(a, \operatorname{liar}(y))$,

$\operatorname{Bel}(b, \operatorname{good}(x)), \operatorname{Bel}(b, \operatorname{liar}(y))$.

All Bel's can be taken off in (20), resulting in

(21) $\neg x=y$, Sato $(x)$, Sato $(y)$, $\operatorname{LiD}(x), \operatorname{AiD}(y), \forall x(\operatorname{liar}(x) \rightarrow \neg \operatorname{good}(x))$, $\operatorname{good}(x), \operatorname{liar}(y)$,

which is shared by $a$ and $b$.

\subsection{Indirect solution of conflicts}

Consider the following dialogue.
(22) 1.a: Today's meeting is held at 203, isn't it? 2.b: No, I heard it is at the small conference room.

3.b: Who told you that?

4.a: Sato told me yesterday.

$5 . b$ : That's strange. I'll call the office.

6.b: They say it was changed from 203 to the small conference room today.

7.a: I sec.

The inference of $(22)$ is formalized as follows:

(23) 1. Sato

2. Sato $\rightarrow 203$

3. 203

1,2

4. office

5. office $\rightarrow$ s.c.r

6. s.c.r

4,5

7. s.c.r $\rightarrow \neg 203$

8. $\neg 203$

6,7

9 .

3,8

In this case, the conflict between (22.1) and (22.2) cannot be solved between themselves. (22.3) to (22.6) reflects the process of deciding which is to be preferred by tracing the source of each condition. That is, when one cannot choose between two conflicting conditions $p_{1}$ and $p_{2}$ on their own account, one replaces $p_{1}$ and $p_{2}$ by $q_{1}, q_{1} \rightarrow p_{1}$ and $q_{2}, q_{2} \rightarrow p_{2}$, respectively, and decide which of $q_{1}, q_{2}$ is to be preferred so that one can avoid the conflict by retracting the weaker condition in favor of the stronger.

\subsection{Conflicts ending in a draw}

Consider the following case.

(24) 1.a: That's Muranishi over there. 2.b: No, it's Hoknto. 3.a: Really?

This case is formalized as follows:

(25) $1, x=y$

2. Hokuto (y)

3. Hokuto $(x)$

4. Muranishi(x)

5. $\forall x($ Muranishi $(x) \rightarrow \neg$ Hokuto $(x))$

6. ᄀHokuto $(x)$

4,5

7.

3,6

As (24.3) indicates, there is no retractable belief in $\mathrm{DB}(K)$, which caused the dialog to end in a breakdown.

\subsection{Formalization of diachronic analy- sis}

The processes of belief revision illustrated in 3.1 through 3.3 can be formalized as in (27). First, we define some terms: 
(26) i) Let $\alpha$ be one of the participants $a$ and $b$ in a dialogue, and $\beta$ the other.

ii) Given $p$ in $\mathbf{D B}(K)$, substitute $B e l(\alpha, p)$ and $\operatorname{Bel}(\beta, p)$ for it. When $\operatorname{Bel}(\alpha, p)$ is replaced by $\operatorname{Bel}(\alpha,-\neg)$, it is called $p$ 's self-denial by $\alpha$. When $\operatorname{Bel}(\alpha, p)$ is simply retracted, it is called $p$ 's selfwithelrawal by $\alpha$.

iii) When $\operatorname{Bel}(\alpha, p), \operatorname{Bel}(\beta, p)$, and $p$ are substituted for by $\neg p$, it is called $p$ 's strong-denial. When they are simply retracted, it is called $p$ 's strong-withdrawal.

iv) Let $\Sigma$ be a set of Horn-clauses, $P I(\Sigma)$ the set of its prime implicants. When $\neg p \in$ $\left\{\neg p_{1}, \ldots, \neg p_{n}\right\}$ for any $q \vee \neg p_{1} \vee \ldots \vee \neg p_{n} \in P I(\Sigma)$, $q$ is subordinate to $p$.

(27) Whenever a now condition is added to $\mathrm{DB}(K)$ in response to a dialogue move, the participant $\alpha$ starts her CMS, calculates a way of resolving any conflict, and revises $\mathrm{DB}(K)$ dynamically: 1) a) When a condition is explicitly registered in DB $(K)$, strip off its belief operator (if any), add it to CMS as an atomic formula.

b) Add implicitly assumed conditionals such as $\forall x($ Muranishi $(x) \rightarrow \neg$ Hokuto $(x))$ to CMS as an atomic formula.

c) Add the implicit inference rules in the dialogue to CMS as a conditional formula. (E.g., the inference rule $a, b / c$ corresponds to the conditional formula $c+a, b$.)

2) Let $\Sigma$ be the set of CMS-clauses obtained in 1). Change $\Sigma$ into $P I(\Sigma)$ (the set of its prime implicants).

a) If $P I(\Sigma) \nvdash \square$, then the dialogue succeds. Lither terminate it, or go on to another.

b) If $P I(\Sigma) \vdash \square$, unless there is a retractable or deniable assumption $p$ in $\Sigma$, go to s). If there is, try to make either p's strong-denial or strongwithdrawal. If it fails, go to c). If successful, for all $q$ such that $q$ is subordinate to $p$, make $q$ 's self-withdrawal, and call the result $\Sigma^{\prime}$.

A) If $P I\left(\Sigma^{\prime}\right) \forall \square$, then the dialogue suceeds. Fither terminate it, or go on to another.

B)If $P I\left(\Sigma^{\prime}\right) \vdash \square$, then $\Sigma:=\Sigma^{\prime}$ and go to b).

c) If every assumption $p$ in $\Sigma$ is well justified, the dialogue fails. If any $p$ has negotiable justifications $q_{1}, \ldots, q_{n}$, replace $p$ by $p$ t. $q_{1}, \ldots, q_{n} ; q_{1}, \ldots, q_{n}$ and call the result $\Sigma^{\prime}$. Set $\Sigma:=\Sigma^{\prime}$, and go to $\left.b\right)$.

\section{Synchronic analysis of dia- logue}

Next, according to Ogata(1993), we consider a classification which characterizes the degree of beliefsharing for the participants at a particular point of the conversation, and the correctness of the shared belicfs.
(28) 1) The belicfs arc all shared by the participants: see (2), (6) above. DB $(K)$ contains no conditions prefixed with Bel. Since the set of beliefs of either participant is considered to be consistent, $I I(2) \forall \square$ for the CMS corresponding to the DRS

2) There remain some conditions prefixed with $B e l$ in DB $(K)$, but $P I(2) \forall \square$ for the CMS corresponding to the DRS. A typical case is when $\beta$ 's assertions properly include $\alpha$ 's belief's and about the rest of $\beta$ 's assertions $\alpha$ has not been able to decide in one way or another.

3) There remain some conditions prefixed with Bel in $\operatorname{DB}(K)$, and $P I(\Sigma) \vdash \square$. This is a case of breakdown as seen in (25).

We call these three cases, respectively, 1) observationally susscessful, 2) observationally consistent, and 3) observationally unsuccessful.

Take the case of (2), (6) again. The dialogue was successfully terminated because the Sato a had in mincl and the Sato $b$ had in mind were both students. But suppose a's Sato was a student in the linguistics department, and b's Sato in the AI repartment, that is, they were different persons. Or suppose $a$ and $b$ had the same Sato in mind, but that he was no longer a stuclent at the time. These two cases are observationally successful, but the participants end up with the wrong beliefs. In order to meet this gap, we introduce a standard of correctness that might be embodied by God's viewpoint, the rcality, or the conventions of the language community to which the participants belong. We call this standard the facts. The categories in (28) are further broken clown relative to the facts as in (29):

Define $K^{\prime}$ as the result of adding the facts to the DB $(K)$ of a DIRS $K$, and extending $\mathbf{U}_{K}$ accordingly. Let $P^{\prime} I\left(\Sigma^{\prime}\right)$ be the set of prime inplicants for the CMS corresponding to $\mathrm{DB}\left(K^{\prime}\right)$. The facts are a set of bare formulas. Then (28) is subclassified as follows:

(29) 1) observationally successful
a) $\operatorname{PI}\left(\Sigma^{\prime}\right) \forall \square$
b) $\operatorname{P} I\left(\Sigma^{\prime}\right) \vdash \square$.
2) observationally consistent
a) $\operatorname{PI}\left(\Sigma^{\prime}\right) \forall \square$
b) $P I\left(\Sigma^{\prime}\right) \vdash \square$.
3) observationally unsuceessful
$P I\left(\Sigma^{\prime}\right) \vdash \square$.

We (all $1 a)$ strongly successful, 2a) strongly consistent, and the rest (the cases where $P I\left(Z^{U}\right) \vdash \square$ ) strongly unsuccessful. A comparison of (28) and (29) suggests the following implications whose converses do not hold:

(30) a) strongly succossful $\rightarrow$ observationally success ful

b) observationally unsuccessful $\rightarrow$ strongly unsuccessful

c) strongly consistent $\rightarrow$ observationally consistent: 


\subsection{Characterization of expressions referring to individuals}

We consider the problem of how the concepts of success introduced in the previous section might be applied to the dialogues identifying the denotation of individual terms, especially proper nouns.

(31) $a$ : That's Sato over there.

$b$ : Yes, it is.

$\mathrm{DB}(K)$ for $(31)$ is

(32) a) $x=y$,

b) $\operatorname{Sato}(x), \operatorname{Sato}(y)$.

If $a$ and $b$ believe there is only one Sato in this situation, (32b) becomes

(33) $\operatorname{Bel}(a, \iota x . \operatorname{Bel}(a, \operatorname{Sato}(x))=x)$, $\operatorname{Bel}(b, \iota x \cdot \operatorname{Bel}(a, \operatorname{Sato}(x))=x)$, $\operatorname{Bel}(a$, tx.Bel $(b, \operatorname{Sato}(x))=y)$, $\operatorname{Bel}(b, \iota x \cdot \operatorname{Bel}(b, \operatorname{Sato}(x))=y)$,

which gives rise to

(34) $\iota x . B e l(a, \operatorname{Sato}(x))=x$ ıx.Bel $(b, \operatorname{Sato}(x))=y$.

From this, we obtain by (32a)

(35) $\iota x . \operatorname{Bel}(a, \operatorname{Sato}(x))=\iota x \cdot \operatorname{Bel}(b, \operatorname{Sato}(x))$.

If (31) is a case of strong success in which "Sato" correctly refers to the unique Sato, $\mathbf{D B}\left(K^{\prime}\right)$ contains

(36) $x=z, \iota x . \operatorname{Sato}(x)=z$.

From (32a), (34), and (36), we can derive

(37) $\operatorname{tx} . \operatorname{Sato}(x)=$ $\iota x \cdot \operatorname{Bel}(a, \operatorname{Sato}(x))=\iota x \cdot \operatorname{Bel}(b, \operatorname{Sato}(x))$.

In general, of an atomic formula $T(x)$, we call $\iota x . B e l(a, T(x))$ a's intended referent, $\iota x . B e l(b, T(x))$ $b$ 's intended referent, and $\iota x . T(x)$ the semanitc referent. Thus, a strongly successful dialogue with regard to an identification of an individual referent is a case where $a$ 's intended referent, $b$ 's intended referent, and the semantic referent all coincide.

However, if in (31) the individual referred to is Kinoshita rather than Sato, $\mathrm{DB}\left(K^{\prime}\right)$ will contain

(38) $\neg(z=x)$, เx.Sato $(x)=z$.

If $a$ and $b$ have different Sato's in mind, $\mathbf{D B}\left(K^{\prime}\right)$ will contain

(39) $\iota x . \operatorname{Sato}(x)=\dagger, \neg(\dagger=x)$.

In either case, the result is strongly unsuccessful:

(40) $\neg(\iota x . \operatorname{Sato}(x)=\iota x \cdot \operatorname{Bel}(a, \operatorname{Sato}(x)))$, $\neg(\iota x \cdot \operatorname{Sato}(x)=\iota x \cdot \operatorname{Bel}(b$, Sato $(x)))$.

A case of being observationally unsuccessful such as (24) will be
(41) $\neg(\iota x \cdot \operatorname{Bel}(a, \operatorname{Sato}(x))=\iota x \cdot \operatorname{Bel}(b, \operatorname{Sato}(x)))$.

By indicating $a$ 's intended referent, $b$ 's intended referent, and the semantic referent by $\mathbf{T a}, \mathbf{T b}$, and $\mathbf{T c o m}$, respectively, we can summarize what has been discussed above as follows:

(42) strongly successful: $\mathbf{T c o m}=\mathbf{T a}=\mathbf{T} \mathbf{b}$, observationally successful: $\mathbf{T a}=\mathbf{T b}$, strongly unsuccessful: Tcom $\neq \mathbf{T a}$, Tcom $\neq \mathbf{T b}$, observationally unsuccessful: $\mathbf{T a} \neq \mathbf{T} \mathbf{b}$.

\section{Conclusion}

In this paper, we proposed a system which combines DRS with CMS, and an algorithm for the dynamic revision of shared beliefs in cooperative dialogues. Further, the degree of success in dialogues was formalized.

Still, the following problems remain to be solved:

1) The treatment of background knowledge must be made precise. E.g., 'Sato $\rightarrow$ 203' in (23), or ' $\forall x($ Muranishi $(x) \rightarrow \neg$ Hokuto $(x))$ ' in $(25)$ is implicitely introduced into the inference without explanation of its origin.

2) The translation proccdure of an utterance into the condition of DRS must be formalized.

3) It's necessary to give a semantic foundation to our system.

4) Implementation of a system which simulates our dialoģue mechanism.

\section{References}

[1] Appelt, D. (1985). "Planning English referring cxpressions". Artificial Intelligence, 26, 1-33.

[2] Asher, N. (1993). Reference to Abstract Objects in Discourse, Dordrecht: Kluwer.

[3] Clark, H. \& Marshall, C. R. (1981). "Definite reference and mutual knowledge". In Joshi et al. Ed., Elements of Discourse Understanding, Cambridge University Press.

[4] Kleer, J. de (1986). "An assumption-based TMS". Artificial Intelligence, 28, 127-162.

[5] Doyle, J. (1979). "A truth maintenance system". Artificial Intelligence, 12, 231-272.

[6] Gärdenfors, P. (1988). Knowledge in flux: modeling the dynamics of epistemic states, Cambridge: The MIT Press.

[7] Joshi, Aravind K. (1982), "Mutual Beliefs in Question-Answer Systems". In Smith, N. V. Ed., Mutual Knowledge, pp. 181-197, London: Academic Press. 
[8] Kanp, H. (1981). "A Theory of trath and semalttic representation". Tn Groenendijk et al. $F d$., Formal Methods in the Study of language, p1). 277-322, Amsterdan: Mathematiseh Centrum Tracts.

[9] Kamp, H. (1990). "Prolegomena to a Structural Account of Belief and Other Attitudes". In Anderson, C. A. \& Owens, J. l'dl., Propositional Attitudes: The Role of Content in Logic, Ianguage, and Mind, pp. 27-90, Stanford: CSII.

[10] Levesque, H. J. (1989). "A knowledge-level account of abduction". IJCAI-89, 1061-1068.

[11] Nadathur \& Joshi (1983). "Mutual belief in conversational systems: their role in referring $\mathrm{ex}$ pressions". IJCAI-83, 603-605, Karlsruhe.

[12] Ogata, N. (1993). "Koyümei, Shiji, Jōhö Kyōyü" "Proper Names, Reference, and InformationSharing"). Sofuto-uea Bunsho no tameno Nihongo Shori no Kenkyii (Study of Japunese? P'rocessing for Software Documents), 12, 257310, Tokyo: Information-Technology Promotion Agency (IPA).

[13] Perranlt, C. R. \& Cohen, P. R. (1981). "It's for your owil good: a note on inaccurate reforence". In Josti et al. Ed., Illements of Discourse Understanding, Cambridge University Press.

[14] Poole, D. (1988). " $\Lambda$ methodology for using a default and abductive reasoning system." Technical report. Dept. of Computer Science. Univ. of Watcrloo, Waterloo.

[15] Reggia, J. (1983). "Diagnostic: expert systems based on a set-covering model". International Journal of Man Machine Studies, 19(5), 437-460.

[16] Reiter, R. \& Kleer, J. de (1987). "Foundations of assumption-based truth maintenance systems: preliminary report". AAAI-87, 183-188, Seattle, Wh. 